\title{
Electron Energy-Loss Spectroscopic Imaging for Phase Detection in Organic Photovoltaics
}

\author{
Ondrej Dyck ${ }^{1}$, Sheng $\mathrm{Hu}^{2}$, Bamin Khomami $^{2}$, and Gerd Duscher ${ }^{1}$ \\ 1. Materials Science and Engineering, University of Tennessee, Knoxville, TN, USA \\ 2. Chemical and Biomolecular Engineering, University of Tennessee, Knoxville, TN, USA
}

Recent years have seen an ever increasing interest in organic photovoltaics (OPV) [1]. As a result there is a high scientific demand for microstructural characterization of the various fabricated devices. Here we introduce several new techniques for examining OPV materials in a (scanning) transmission electron microscope (TEM/STEM) capable of electron energy-loss spectroscopy (EELS). The materials under investigation are regio-regular poly(3-hexylthiophene) (P3HT) and [6,6]-phenyl $\mathrm{C}_{61}$-butyric acid methyl ester (PCBM) which form the most commonly used donor/acceptor blend[1].

A common method for producing phase contrast in the TEM is by introducing a post-specimen energy window that only allows electrons having a specific range of energies form the image [2-6]. This is known as energy filtered TEM (EFTEM). This technique produces contrast of phases but it is a qualitative measurement, only. The underlying mechanism that allows it to work is bulk pasmon energy shifts in the electron energy loss (EEL) spectrum. However, these shifts are not quantitatively mapped across the sample. We present quantitatively mapped plasmon peak positions across a P3HT/PCBM bulk heterojunction. In conjunction we also mapped absolute carbon density across the same area. P3HT is less carbon dense than PCBM and thus, the difference in carbon concentration is visible in the carbon density map [7]. Furthermore carbon density may also be used to produce a quantitative map of $\mathrm{P} 3 \mathrm{HT} / \mathrm{PCBM}$ ratio for a much more precise understanding of the BHJ structure.

EFTEM imaging was performed to distinguish between P3HT and PCBM for comparison to the EELS imaging results. Figure 1 (left) represents a typical EFTEM image from the sample. EELS spectrum images were also taken of the cross-section samples. With this technique the electron beam is rastered across the sample and an EEL spectrum recorded at each position (Figure 1 middle). Movement of the plasmon peak is responsible for the intensity variations highlighted in the EFTEM image (Figure 1 left). The color in Figure 2 (left) shows the energy position of peak 2 while the $x$ and $y$ dimensions represent physical position on the sample. This measurement seems to indicate a PCBM-rich layer at the surface of the sample as seen in Figure 2 (left). For each core-loss spectrum, a corresponding low-loss spectrum was also acquired. The low-loss data was fit with a series of Lorentzian peaks to obtain a noise free model low-loss. Each core-loss spectra was background-subtracted and fit with a hydrogenic cross-section which had been convoluted with the low-loss model. Carbon areal density was then calculated based on scattering probability. The result of this analysis is shown in Figure 2 (right) where the color represents carbon areal density.

The similarity between Figures 2 (left) and 2 (right) is clear. There is a higher carbon density in the same locations where the plasmon peak shifts to higher energy. The only area in which they disagree is the surface layer. The carbon density map shows low carbon density indicating little PCBM while the plasmon peak position map anomalously shows a PCBM-like shift. This layer is actually the platinum layer deposited during the FIB sample preparation, which has a bulk plasmon energy close to PCBM. 
To produce a P3HT/PCBM ratio map the thickness of the sample must be known. To estimate the sample thickness, the carbon areal density measurements were compared to known carbon densities for these materials. Since carbon areal density varies with thickness a thickness measurement can be obtained. Additionally a series of carbon areal density measurements were acquired at various tilt angles to check for self-consistency as sample thickness changed. We see measurements that are self-consistent to within a couple nanometers (figure 1 right). Once thickness is established, a linear combination of P3HT and PCBM carbon densities can be used to replicate the observed carbon signal and produce a phase ratio map of the sample. This allows precise identification of composite phases that are very difficult to reliably distinguish with other methods. This technique can easily be extended to any carbon-based blend of materials that exhibit differing carbon concentrations.

\section{References:}

[1] M. T. Dang, L. Hirsch, G. Wantz, Advanced Materials 23 (2011), p. 3597-4095.

[2] P. G. Nicholson, F. A. Castro, Nanotechnology 21 (2010), p. 492001.

[3] A. A. Herzing, L. J. Richter, I. M. Anderson, The Journal of Physical Chemistry C 114 (2010), p. 17501-17508.

[4] L. F. Drummy et al., Chemistry of Materials 23 (2011), p. 907-912.

[5] M. Pfannmöller et al., Nano Letters 11 (2011), p. 3099-3107.

[6] W. Schindler, M. Wollgarten, K. Fostiropoulos, Organic Electronics 13 (2012), p. 1100-1104.

[7] A. A. Herzing et al., ACS Nano 7 (2013), p. 7937-7944.

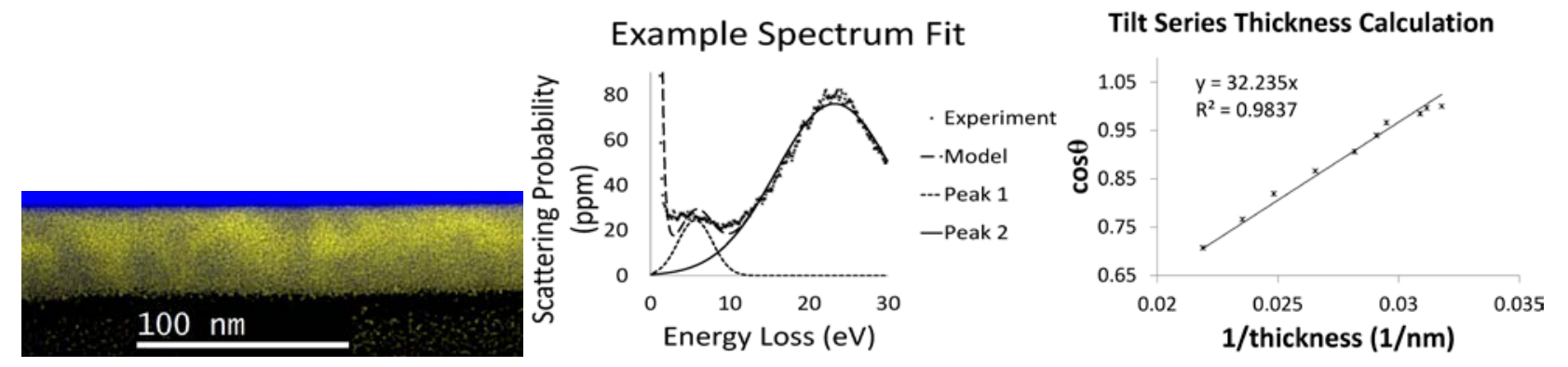

Figure 1. Left: EFTEM composite image showing contrast between P3HT (dark yellow) and PCBM (bright yellow). The Si substrate appears blue due to the strong plasmon in the lower energy range. Middle: Plot shows an example low-loss EEL spectrum fit with two gaussian curves which are used to precisely measure the position of the plasmon peak. Right: A carbon density tilt series shows thickness measurements consistent with carbon density thickness measurements.
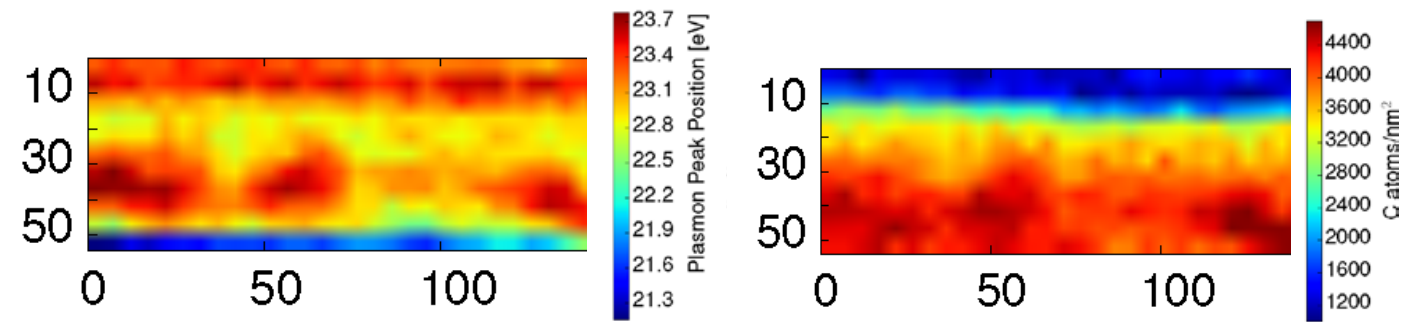

Figure 2. Left: Plasmon peak position map across the active region of the device. Red indicates higher PCBM concentration. Right: Carbon density mapping exhibits similar features, indicating that carbon density may also be used to determine phase location. The data disagree at the top surface where a layer of Pt introduces anomalous results for the plasmon peak fitting routine. Lack of carbon conclusively shows no agglomeration of PCBM at the top surface. 